\title{
Characterization of Stra8 in Southern catfish (Silurus meridionalis): evidence for its role in meiotic initiation
}

\author{
Ranran Dong, Shijie Yang, Jing Jiao, Tingru Wang, Hongjuan Shi, Linyan Zhou, Yaoguang Zhang \\ and Deshou Wang*
}

\begin{abstract}
Background: RA (retinoic acid) signal pathway has been proved to be required for germ cell meiotic initiation in mammals, aves and amphibians. Stra8 (Stimulated by retinoic acid gene 8) is an important factor in RA signal pathway. However, the role of RA and Stra8 in germ cell meiotic initiation in teleosts is poorly characterized.

Results: In this study, the full length cDNA of Stra8 was cloned from Southern catfish (Silurus meridionalis), and its spatio-temporal expression profiles were analyzed. The Stra8 cDNA (1606 bp) includes 163 bp 5'-UTR (untranslated region), 456 bp 3'-UTR, and an ORF (open reading frame) of 987 bp, encoding a polypeptide of 328 aa.

Phylogenetic analysis revealed its existence in some primitive teleosts, such as Siluriformes and Salmoniformes. Tissue distribution analysis by RT-PCR showed that Stra8 is specifically expressed in gonads. By real-time PCR, in situ hybridization and immunohistochemistry, the highest expression level of Stra8/Stra8 was detected in 50 and 130 dah (day after hatching), the premeiotic stage of germ cells in XX and XY gonads, respectively.
\end{abstract}

Conclusions: Our results suggest that Stra8 might be involved in germ cell meiotic initiation in S. meridionalis as it did in tetrapods.

Keywords: Stra8, mRNA and protein, Expression profile, Meiotic initiation, Silurus meridionalis

\section{Background}

Meiosis is a special type of cell division necessary for sexual reproduction in eukaryotes. It not only generates genetic diversity on which natural selection can act, but also helps maintain the immortality of the germ line [1]. In mammals, meiosis is initiated at different time points in males and females. Recent discoveries indicate that the key to this sex-specific timing of meiosis entry is the presence or absence of the signaling molecule RA (retinoic acid) [2-7]. In mouse, a model was proposed in which RA signaling and metabolism regulate whether female and male germ cells initiate meiosis during embryogenesis. The model posits that, in embryonic ovaries, RA induces germ cells to express Stra8, which in turn leads to meiotic initiation. In embryonic testes,

\footnotetext{
* Correspondence: wdeshou@swu.edu.cn

Key Laboratory of Freshwater Fish Reproduction and Development (Ministry of Education), Key Laboratory of Aquatic Science of Chongqing, School of Life Science, Southwest University, Chongqing, China
}

an enzyme of the Cyp26 family, likely Cyp26b1, degrades RA and thereby prevents expression of Stra8 and precludes meiotic initiation [8-10]. In avians and amphibians, the role of RA in meiotic initiation is similar to that in mammals showing that RA-dependent meiosis entry could be a conserved mechanism of germ cell differentiation in tetrapods $[11,12]$.

Stra8 which was first identified in a screen for genes induced by RA is specifically expressed in mammalian germ cells before their transition from mitosis to meiosis [13-16]. Studies on the teratocarcinoma cells and embryonic stem cells (ESC) transfected with Stra8-EGFP shown that the positive cells may undergo meiosis develop into sperm and generate live offspring mice [16]. In female embryos lacking Stra 8 gene function, the early mitotic development of germ cells is normal, but these cells then fail to undergo premeiotic DNA replication, meiotic chromosome condensation, cohesion, synapsis and recombination [17]. Analysis of Stra8-deficient testes of mice demonstrated

\section{Biomed Central}


that Stra8 is essential for normal progression into meiotic prophase $[4,14,17]$. Above all, Stra8 is the key factor involved in RA signal pathway in the control of germ cell meiosis.

The role of Stra8 in the meiosis of teleost has not yet been reported. Some researchers even suspected that Stra8 may be amniote- or even mammalian-specific, according to Ensemble orthologue prediction (http://www.ensembl.org/ index.html) [18]. In the present study, homologous sequences of Stra 8 were isolated from channel catfish, rainbow trout and salmon in EST database, and Stra8 cDNA was cloned from $S$. meridionalis. The spatial and temporal expression patterns of Stra 8 were examined by RT-PCR, real-time PCR, in situ hybridization and immunohistochemistry. The study shows for the first time that Stra8 is expressed highest in the premeiotic phase of germ cell in S. meridionalis. Our data indicated that Stra8 may be involved in meiotic initiation of $S$. meridionalis.

\section{Results}

Cloning and characterization of S. meridionalis Stra8 cDNA Using degenerated PCR amplification, a cDNA fragment of 642 bp was obtained and was confirmed to be a partial sequence of $S$. meridionalis Stra8 gene by homology search in the GenBank database. The full length cDNA of Stra 8 was successfully isolated by RACE-PCR. Stra 8 cDNA (accession number: KC533813) was 1606 bp in length containing an open reading frame (ORF) of 987 $\mathrm{bp}$, corresponding to 328 amino acid residues and the 5 ' and 3' UTRs of 163 and $483 \mathrm{bp}$, respectively. The predicted molecular mass and pI value of the deduced Stra8 protein were $43 \mathrm{kDa}$ and 4.29 , respectively.

\section{Amino acid sequence alignment}

Alignment of the $S$. meridionalis Stra8 with those from other organisms revealed a low identity, especially in the $\mathrm{N}$-terminal regions (Figure 1). The overall similarity between fish and tetrapods Stra8 is very low, averagely less than $30 \%$. It shared $28 \%$ similarity with $H$. sapiens (NP_872295), 29\% with M. musculus (NP_033318) and M. gallopavo (XP_003202232), 30\% with G. gallus (XP_416179), 27\% with A. carolinensis (XP_003221102), and $23 \%$ with $X$. tropicalis (XP_002941476). However, $S$. meridionalis Stra8 shared $75 \%$ with I. punctatus, and $44 \%$ with O. mykiss, respectively. There are three motifs (depicted as Box 1-3) relatively conserved among all Stra8. Box3 is the most important and conserved signature motif. All fish share the same 12 amino acid sequences in this motif while only 3 out of 12 amino acid residues differ between Stra8 from fish and tetrapods.

\section{Phylogenetic analysis of Stra8}

Phylogenetic analysis of Stra8 from representative cephalochordate, fish, amphibians, reptiles, avians and mammals (Figure 2) produced an NJ-phylogenetic tree that contained three distinct branches. The S. meridionalis Stra8 clustered with those of fish, while sequences from tetrapod and cephalochordate formed a separate cluster, respectively.

\section{Tissue distribution by RT-PCR}

In adult $S$. meridionalis, Stra8 was found to be exclusively expressed in gonads, with much higher expression in the testis compared with the ovary (Figure 3).

\section{Expression profiles of Stra8 in gonads by real-time PCR}

As shown in Figure 4(a), the relative expression of Stra8 mRNA was at the highest level in 50 dah ovaries and 130 dah testes, corresponding to the premeiotic stage of germ cells, and at lower levels in 40 and 60 dah ovaries as well 110 and 150 dah testes.

\section{Localization of Stra8 in gonads}

\section{Morphological observation of gonads by H.E. staining}

To observe the germ cell meiosis in female and male gonads, H.E. staining was performed using ovaries at 40, 50 and 60 dah and testes at 110, 130 and 150 dah. The results showed that ovaries at 40,50 and 60 dah were mainly composed of premeiotic oogonia and primary oocytes, respectively; while testes at 110, 130 and 150 dah, were basically composed of synchronized spermatogonia and primary spermatocytes (Figure $4 \mathrm{~b}$ ). The results also showed that the majority germ cells are in the premeiotic phase in 50 dah ovaries and testes130 dah.

\section{Expression, purification, and Western blot analysis}

Recombinant Stra8 protein with a His-tag at its N terminus was successfully expressed in E. coli. Unpurified and purified recombinant Stra8 proteins were analyzed by SDS-PAGE with Coomassie blue staining (Figure 5). The specificity of the Stra8 antibody was confirmed by Western blotting. Specific bands of $43 \mathrm{kDa}$ corresponding to the calculated molecular weights of the S. meridionalis Stra8 fusion proteins and total proteins extracted from 50 dah ovaries and 130 dah testes were recognized using our own Stra8 antibody (Figure 5).

\section{Cell types of Stra8/Stra8 in gonads by ISH and IHC}

To identify the localization of Stra8/Stra8 in gonads, ISH and IHC was performed using ovaries at 40, 50 and 60 dah and testes at 110, 130 and 150 dah. The results showed that specific signals of Stra8/Stra8 were observed in germ cells in gonads of both sexes. Weak mRNA and protein signals were detected in 40 dah ovaries and 110 dah testes (Figure 4 C, I, O and S). Strong signals were found in premeiotic germ cells in 50 dah ovaries and 130 dah testes (Figure 4 D, J, P and T). The signals became weaker in the 60 dah ovary and 150 dah testes 
(See figure on previous page.)

Figure 1 Alignments of S. meridionalis Stra8 with those of other species. ClustalX and GeneDoc were used to make this figure. The overall similarity among the isolated Stra8 is very low, averagely less than $30 \%$. The three relatively conserved motifs were depicted as Box 1-3. The sources of the sequences are described in the Materials and Methods section. Has, H.sapiens; Bta, B. taurus; Mmu, M. musculus; Rno, R. norvegicus; Gga, G. gallus; Clf, C. lupus familiaris; Omy, O. mykiss; Aca, A. carolinensis; Mga, M. gallopavo; Ipu, I. punetaus; Sme, S. meridionalis.

(Figure $4 \mathrm{E}, \mathrm{K}, \mathrm{Q}$ and $\mathrm{U}$ ). The positive signal corresponds to the brownish color (Figure 4 R). No signal was detected in the negative control without the primary antibody (Figure $4 \mathrm{~V}$ ).

\section{Discussion}

In this study, the full length cDNA of the Stra 8 was cloned and characterized in a teleost, S. meridionalis, for the first time. Stra8 sequences were found to be poorly conserved among vertebrates. According to the phylogenetic tree, Stra8 might be existed in the common ancestor of cephalochordate and vertebrate. It is speculated that like in tetrapods Stra8 may be widespread in teleosts. However, in teleosts, Stra 8 was not found in the genome databases of zebrafish, medaka, fugu, tetraodon, stickleback and Nile tilapia. Stra 8 sequences were only isolated from Siluriformes and Salmoniformes by bioinformatics analysis of available databases. There are two possibilities for this, 1) It is difficult to identify Stra8 from other fish species because of the very low sequence similarity; 2) Stra8 is not included in the databases because of incomplete genome sequencing.

In mammals, Stra 8 was expressed in embryonic ovaries just before meiotic initiation, whereas its expression in testes was first detected after birth [2]. In this study, tissue distribution analysis showed that Stra 8 was specifically expressed in gonads of both sexes of adult $S$. meridionalis. In mouse, moderate levels of Stra 8 transcript in gonocytes and the peak of Stra 8 mRNA expression coincided with the onset of meiosis in postnatal testes. Stra 8 mRNA levels were greatly reduced or absent in germ cells once they entered meiosis by microarrays [19]. Interestingly, in this study real-time PCR showed that Stra 8 was expressed at the highest level in ovaries at 50 dah and testes at 130 dah. In addition, our previous work showed that meiosis of germ cell in S. meridionalis initiated at about 50 dah, whereas meiosis of male germ cells initiated at about 130 dah [20]. This unique expression pattern made us curious to examine the cellular localizations of Stra 8 transcripts and protein in gonads. ISH and IHC analysis revealed that Stra8/Stra8 signals were only observed in germ cells. Consistant with the Real-time PCR results, strongest signals were detected in ovaries at 50 dah and testes at 130 dah, respectively. Furthermore, hemotoxylin-eosin (H.E.) staining verified that the majority of germ cells in 50 dah ovaries and in 130 dah testes were in the premeiotic phase. These results suggest that Stra8 may play an important role in meiotic initiation in S. meridionalis.

It is well document that meiosis is initiated through retinoic acid induction of Stra8 in both male and female germ lines in higher vertebrates [21-25]. There is also evidence for similar role of RA in germ cell meiotic initiation in amphibians [12]. In addition, Cyp26b1, an RA catabolic enzyme, was proved to regulate germ cell fate in a manner common among vertebrates in Japanese

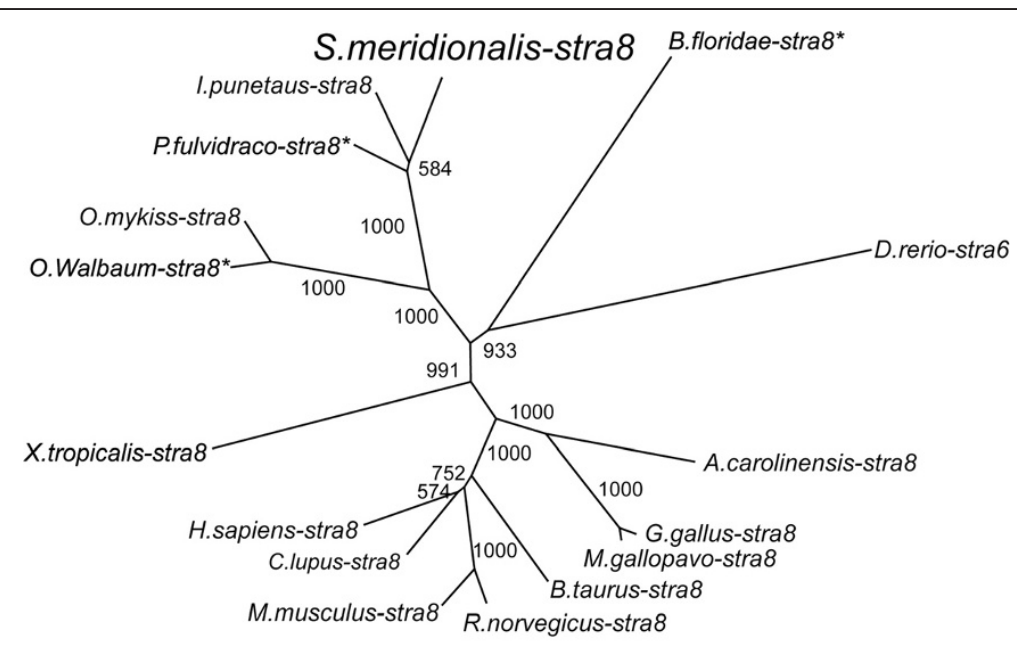

0.1

Figure 2 Phylogenetic tree of Stra8. The tree was made with the default settings of the ClustalX protein alignment program and visualized using treeview32. The values represent bootstrap scores out of 1000 trials, indicating the credibility of each branch. Asterisks indicate partial sequences. 


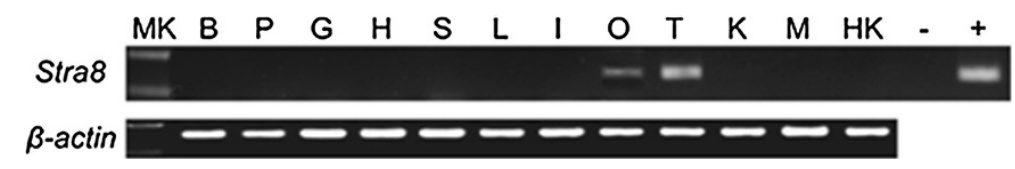

Figure 3 RT-PCR analysis of Stra8 from various adult S.meridionalis tissues. B, brain; P, pituitary; G, gill; H, heart; S, spleen; L, liver; l, intestine; $\mathrm{O}$, ovary; T, testis; K, kidney; M, muscle; HK, head kidney; +, positive control; -, negative control. Lower panel: $\beta$-actin was used as internal control.

flounder [26]. Long term treatment of XX Nile tilapia with Citral, an RA synthetase inhibitor, from 5 dah resulted in female to male sex reversal (unpublished data from our group). In the present study, we showed evidence for possible involvement of Stra8, the key factor for RA signal pathway, in germ cell meiotic initiation in $S$. meridionalis. Taken together, these data strongly suggest that RA may play important roles in germ cell meiotic initiation in teleosts as it did in tetrapods.

\section{Conclusions}

Stra8 cDNA full-length was successfully cloned from S. meridionalis. Sequence and phylogenetic analyses demonstrated that it is the genuine counterpart of mammalian Stra8. Tissue distribution analysis revealed that it was exclusively expressed in the gonad. Real-time PCR, ISH and IHC analyses showed that it was highly expressed in the premeiotic phase of germ cells in both ovaries and testes. These results indicated that Stra8 probably participated in meiotic initiation in S. meridionalis as it did in higher vertebrates. Stra8 is the key factor in RA induced germ cell meiotic initiation. Together with the results from Japanese flounder and tetrapods, we conclude that RA-dependent meiotic initiation might be a conserved mechanism of germ cell differentiation in vertebrates.

\section{Methods}

Animals

Adult S. meridionalis which has XY heterogametic sex determination were obtained from the Jialing River, a branch of the Yangtze River, and were kept in aerated tanks until use. All fry used in the experiments were obtained by artificial propagation using the same parental fish raised in our laboratory. All animal experiments conformed

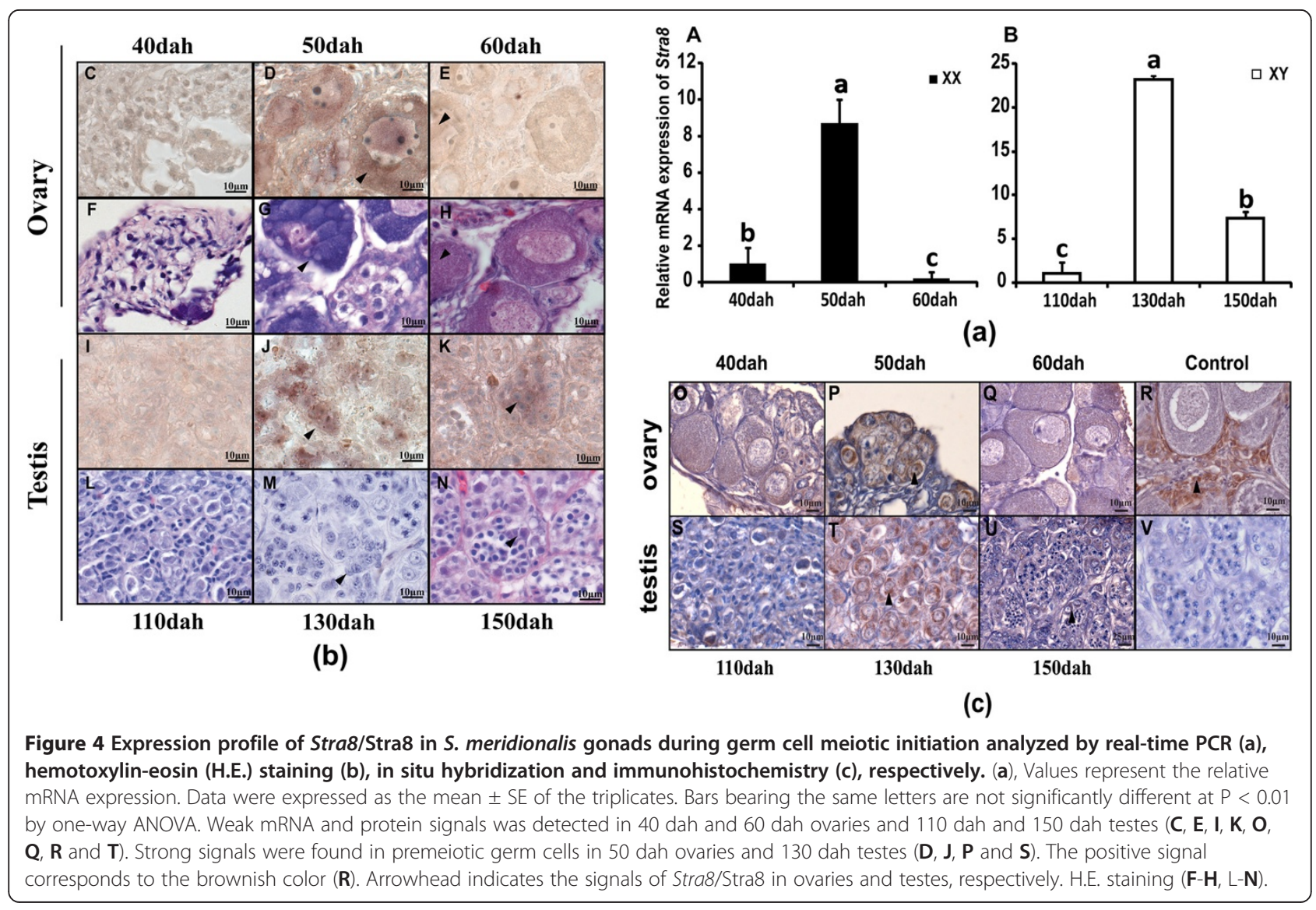




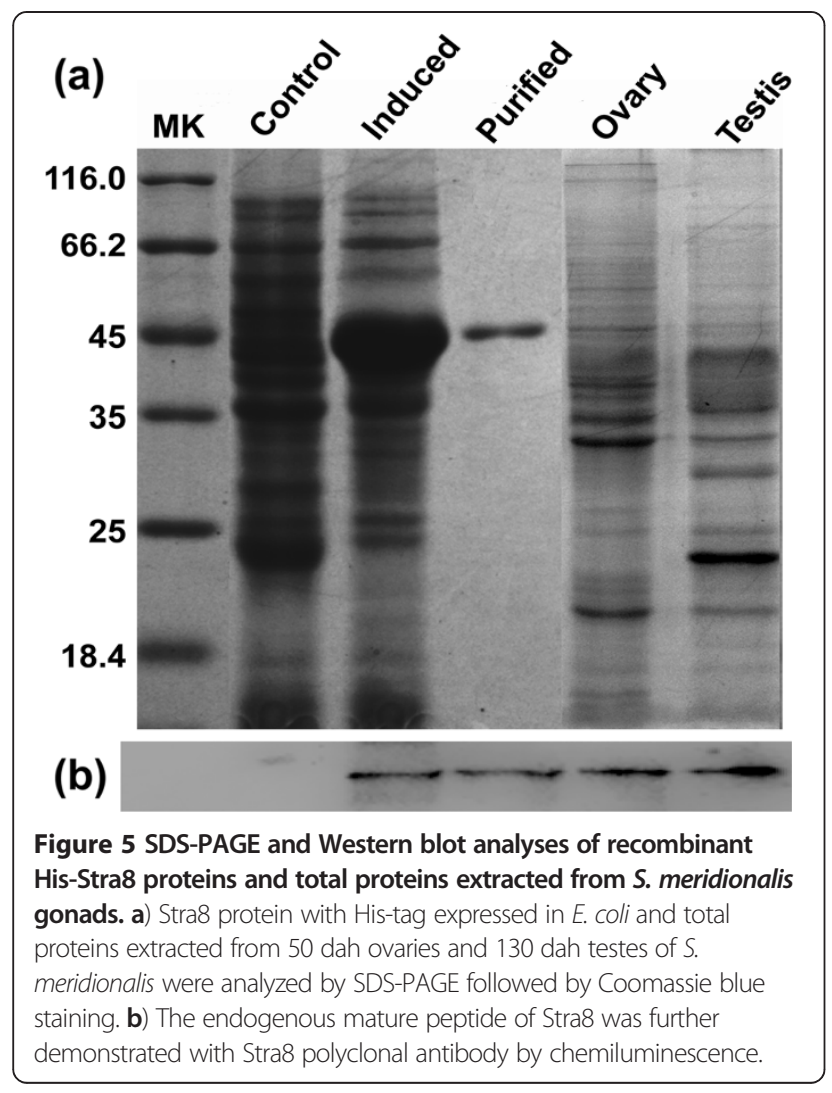

to the Guide for Care and Use of Laboratory Animals and were approved by the Committee of Laboratory Animal Experimentation at Southwest University, Chongqing, China.

The pre-meiotic, meiotic and post-meiotic stages of germ cells were assessed according to meiotic initiation time and germ cell morphology characterized in S. meridionalis [20]. Gonads were sampled at these stages for real-time PCR, H.E., ISH and ICH staining.

\section{Nucleic acid preparation and first-strand CDNA synthesis}

Total RNA was extracted from various tissues using Tissue RNA Rapid Extraction Kit (TaKaRa, Japan) according to the manufacturer's protocol. The quantity and quality of RNA were examined by UV-spectrophotometry (OD260/OD280) and agarose gel electrophoresis, respectively. Total RNA (500 ng) from various tissues were reverse transcribed into first-strand cDNA using PrimeScript RT Master Mix Perfect Real Time Kit (TaKaRa, Japan) according to the manufacturer's instructions.

\section{Degenerated RT-PCR (reverse transcription polymerase chain reaction)}

Homologous sequences of Stra8 were isolated from Channel Catfish, rainbow trout and salmon in EST database. However, Stra 8 was not found in the genome databases of zebrafish, medaka, fugu, tetraodon, stickleback and Nile tilapia. Degenerated primers (Table 1) were designed based on the conserved domain from known Stra 8 homology sequences and were used to amplify the target fragment of the Stra8 cDNA. First strand cDNA was subjected to PCR amplification using Premix Ex $\mathrm{Taq}^{\mathrm{Tm}}$ Hot Start Version (TaKaRa, Japan) and degenerated primers $(10 \mu \mathrm{M})$ were used at final concentrations in a $25 \mu \mathrm{l}$ reaction for $94^{\circ} \mathrm{C}$ for $3 \mathrm{~min}$, followed by 30 cycles of $94^{\circ} \mathrm{C}$ for $30 \mathrm{~s}, 58^{\circ} \mathrm{C}$ for $30 \mathrm{~s}, 72^{\circ} \mathrm{C}$ for $2 \mathrm{~min}$ and a final elongation step at $72^{\circ} \mathrm{C}$ for $8 \mathrm{~min}$. PCR products were separated on 1.5\% agarose gel and purified with an Gel Extraction Kit (Omiga BioTek, USA). The purified PCR products were cloned into a pMD19-T vector (TaKaRa, Japan) overnight. The recombinant plasmid was transformed into E. coli competent cells DH5 $\alpha$ and positive clones were sequenced at Life Technologies Corporation (Shanghai, China). The retrieved sequences were verified and analyzed for similarity with other known Stra 8 sequences using the BLASTX program at the National Center for Biotechnology Information (http://www.ncbi.nlm.nih.gov/blast.cgi).

\section{Rapid amplification of CDNA ends (RACE)}

Full-length Stra 8 cDNA was obtained by 5'- and 3'-RACE method using the SMARTer ${ }^{\mathrm{m}}$ RACE cDNA Amplification Kit (Clontech, USA) according to the manufacturer's protocol. The 5'- and 3'-RACE primers (Table 1) were designed based on the obtained Stra 8 cDNA fragment sequences. The PCR program was performed for 26 cycles of $94^{\circ} \mathrm{C}$ for $30 \mathrm{~s}, 68^{\circ} \mathrm{C}$ for $30 \mathrm{~s}$ and $72^{\circ} \mathrm{C}$ for $3 \mathrm{~min}$. The amplified cDNA fragments were cloned and sequenced as described for degenerated RT-PCR above. Then the full-length Stra 8 cDNA was sequenced again to confirm the nucleotide sequences.

\section{Multiple sequence alignment and phylogenetic analysis}

Deduced protein sequences of Stra8 from various species were retrieved from GenBank and phylogenetically compared with those of $S$. meridionalis. Multiple alignments were carried out using ClustalX and GeneDoc [27]. A bootstrapped neighbor-joining phylogenetic tree using $D$. rerio Stra6 (NP_001038777) as outgroup was constructed and viewed with Treeview 32. The credibility of the branching was tested using bootstrap resampling with 1000 pseudo replicates. Except S. meridionalis Stra8 which was cloned in this study, all other Stra8 protein sequences were obtained from the NCBI database (http://www. ncbi.nlm.nih.gov/). The accession numbers of these Stra8 protein sequences are: $H$. sapiens (NP_872295), $C$. familiaris (XP_852820), M. musculus (NP_033318), $R$. norvegicus (XP_575429), B. Taurus (XP_001253650), M. gallopavo (XP_003202232), G. gallus (XP_416179), A. carolinensis (XP_003221102), X. tropicalis (XP_002941476), O. mykiss (CX037430), S. salar (DW470925). 
Table 1 Primers used in the present study

\begin{tabular}{|c|c|c|}
\hline Primer name & Primer sequence $\left(5^{\prime}\right.$ to $\left.3^{\prime}\right)$ & Purpose \\
\hline Stra8- $\mathrm{F}_{1}$ & CCAACATCCAGGAGTTTGA & CDNA fragment PCR \\
\hline Stra8- $\mathrm{F}_{2}$ & GGGATATCTCTITTTCTAC & \\
\hline Stra8- $\mathrm{F}_{3}$ & GCCATCTCAGGCCTGTGG & \\
\hline $\mathrm{Stra8}-\mathrm{R}_{3}$ & CCTGAGATGGCCTCGGACACTAC & \\
\hline Stra8- $R_{2}$ & CTGTAGTAGATCCTCCTCA & \\
\hline Stra8- $R_{2}$ & CACCACATCATAGGCATCCTG & \\
\hline Stra8-RACE- $F_{1}$ & GAGCTTCTCTTGCGCAGTGGAGTG & RACE PCR \\
\hline Stra8-RACE- $F_{2}$ & GTCCGAGGCCATCTCAGGCCTGTGGA & \\
\hline Stra8-RACE- $\mathrm{F}_{3}$ & CCACTGACTCACATCTCTACGGCTT & \\
\hline Stra8-RACE-R $\mathrm{R}_{3}$ & CACTCCACTGCGCAAGAGAAGCTCGA & \\
\hline Stra8-RACE- $R_{2}$ & CACAGGCCTGAGATGGCCTCGGACACT & \\
\hline Stra8-RACE- $\mathrm{R}_{1}$ & GCCGTAGAGATGTGAGTCAGTGGTCT & \\
\hline Stra8-sF $F_{0}$ & CCTCAGCAAGCTCCTTGCACTGAA & Tissue distribution \\
\hline Stra8-sRo & GCTCTGCAGTACTGAAGGCCTGTT & \\
\hline Stra8- $F_{0}$ & GAGCAGGTATGTCCCGTTGG & Full length cDNA amplification \\
\hline Stra8- $\mathrm{R}_{0}$ & GGAGGAACTGGCTAGAAATCC & \\
\hline Stra8-Q-F & AGTGCCTGATGTAGATGA & Real-time PCR \\
\hline Stra8-Q-R & AGAGTCTCGCTGTAGAAG & \\
\hline sc- $\beta$-actin- $F$ & GGCATCACACCTTCTACAACGA & Internal control \\
\hline$s c-\beta$-actin-R & ACGCTCTGTCAGGATCTTCA & \\
\hline
\end{tabular}

\section{Tissue distribution by RT-PCR}

Gene-specific primers were used for the RT-PCR analysis. Positive and negative controls were set up with plasmid DNA and water, respectively, as templates to validate the distribution pattern. A $342 \mathrm{bp}$ fragment of $\beta$-actin was amplified (as internal control) from $S$. meridionalis to test the quality of the cDNAs used in the PCR. The PCR conditions consisted of $94^{\circ} \mathrm{C}$ ( $3 \mathrm{~min}$ ), followed by 30 cycles of $94^{\circ} \mathrm{C}(30 \mathrm{~s}), 62^{\circ} \mathrm{C}(30 \mathrm{~s})$, and $72^{\circ} \mathrm{C}(30 \mathrm{~s})$, the reaction was ended by a further $10 \mathrm{~min}$ at $72^{\circ} \mathrm{C}$. PCR was performed on a C1000 thermal cycler (Bio-Rad, USA). All the PCR products were subjected to agarose gel (1.5\%) electrophoresis and gels were stained with ethidium bromide to visualize bands.

\section{Real-time PCR analysis}

mRNA expression analysis for different meiotic stages of ovaries and testes were conducted via Real-time PCR according to the following method: gene-specific primers (Table 1) were designed based on the cloned Stra 8 cDNA to produce an amplicon of $152 \mathrm{bp}$. Real-timePCR was performed in a $\mathrm{C}_{1000}{ }^{\mathrm{Ts}}$ Thermal Cycler (BioRad CFX 96 ${ }^{\mathrm{Tm}}$ Real-Time System) according to the manufacturer's protocol. The final volume of each Realtime-PCR reaction was $20 \mu \mathrm{l}$, which contained $10 \mu \mathrm{l}$ $2 \times$ SYBR Premix ExTaq (TaKaRa, Japan), $2.0 \mu \mathrm{l}$ of 10 -fold diluted cDNA template, $6 \mu \mathrm{l}$ of PCR-grade water, and
$1.0 \mu \mathrm{l}$ of each $10 \mu \mathrm{M}$ primer. PCR conditions were as follows: $95^{\circ} \mathrm{C}$ for $30 \mathrm{~s}$, followed by 40 cycles of $95^{\circ} \mathrm{C}$ for $5 \mathrm{~s}$ and $60^{\circ} \mathrm{C}$ for $30 \mathrm{~s}$. Each sample was run in triplicates and normalized to the selected control gene $\beta$-actin of S. meridionalis. The primers of $\beta$-actin (Table 1 ) were designed to produce an amplicon of $342 \mathrm{bp}$.

Stra 8 expression levels were calculated by the $2^{-\Delta \Delta C t}$ Comparative CT method [28]. Mean and standard deviations were calculated from triplicate experiments, and presented as $\mathrm{n}$-fold differences in expression relative to $\beta$-actin. Data was analyzed using the the CFX Manager ${ }^{\mathrm{TM}}$ software (version 1.0). Data is reported as mean \pm standard error of mean (S.E.). The homogeneity of variance was confirmed and comparison between means was performed with a one-way ANOVA. Duncan's procedure was used for multiple comparisons between groups. Differences were regarded as significant when $\mathrm{P}<0.01$. All statistical analyses were performed by STATISTICA 6.0.

\section{Hemotoxylin-eosin (H.E.) staining and in situ hybridization (ISH)}

Gonads were fixed in Bouin's solution, embedded in paraffin, and cross-sectioned at $5 \mu \mathrm{m}$. Sections were deparaffinized, hydrated, H.E. stained and mounted.

Gonads were fixed in $4 \%$ paraformaldehyde in $0.85 \times$ PBS $(\mathrm{pH} 7.4)$ at $4^{\circ} \mathrm{C}$. After fixation, gonads were embedded in paraffin. Cross sections were cut at $5 \mu \mathrm{m}$. Probes of 
sense and anti-sense digoxigenin-labeled RNA strands were transcribed in vitro with an RNA labelling kit (Roche, Germany) from plasmid DNA containing ORF of the Stra8. The hybridization was carried out as follows: sections $(5 \mu \mathrm{m})$ were deparaffinized, hydrated and treated with proteinase K (Amersco, USA.) and then hybridized with the sense or antisense DIG-labelled RNA probe at $60^{\circ} \mathrm{C}$ for $18-24 \mathrm{~h}$. The hybridization signals were then detected using alkaline phosphatase-conjugated antiDIG antibody (Roche, Germany) and NBT as described previously [29].

\section{Stra8 recombinant protein expression, antibody production and Western blot analysis}

An expression vector of Stra8 was constructed using pCold I. Recombinant S. meridionalis Stra8 with His-tag at its N-terminal was expressed in E. coli by IPTG induction until the absorbance value reached an $\mathrm{OD}_{600}$ of 0.5-0.6. His-Stra8 recombinant protein purified using Ni-NTA superflow cartridge (Qiagen) was used as the antigen to immunize rabbit (female, from Chongqing Medical University Animal Centre) three times at 15 day intervals with 25-30 $\mu \mathrm{g}$ antigen each time for the production of polyclonal antibodies. Ten days after the last immunization, rabbit blood was collected for ELISA evaluation. To confirm the polyclonal antibody specificity, total proteins extracted from both the fish gonads as well as the His-Stra8 recombinant proteins (both purified and unpurified) were separated on 12\% SDSPAGE (sodium dodecyl sulfate-polyacrylamide gel electrophoresis) gels. The separated proteins were transferred onto PVDF membranes and immunoblotted with the primary antibodies against Stra8 (Anti-Stra8, ×1000). After PBS washing, chemiluminescence was applied for the detection after incubation with secondary antibody conjugated with horseradish peroxidase. In order to enhance the signal of Stra8 protein from fish gonads, chemiluminescence was performed as described previously [5], and the signal was visualized on Fusion Fx (BioRad, USA) using super signal west picoluminol /Enhancer Solution and super signal west picostable peroxide Solution (Pierce, USA) as substrates after PBS washing.

\section{Immunohistochemistry (IHC)}

After washing with $0.01 \mathrm{M}$ PBS three times for $10 \mathrm{~min}$ per wash, sections were immersed in $0.01 \mathrm{M}$ citric acid buffer (pH 6.0) containing $0.1 \%$ Tween 20 , and autoclaved for $5 \mathrm{~min}$. The sections were then treated in a blocking solution (Roche, China), incubated with Stra8 rabbit polyclonal antibody $(1: 1000)$ overnight at $4^{\circ} \mathrm{C}$, and rinsed with $0.01 \mathrm{M}$ PBS three times for 5 min per wash. Subsequently, the tissue sections were incubated with a second antibody (goat anti-rabbit IgG) conjugated with horseradish peroxidase (Bio-Rad) at 1:2000 for $30 \mathrm{~min}$, and then rinsed with PBS three times for 5 min per wash. Immunoreactive signals were visualized using diaminobenzidine (Sigma) as the substrate. Sections were counterstained with hematoxylin. For the negative control, the primary antibody was replaced with normal rabbit serum.

\section{Competing interests}

The authors declare that they have no competing interests.

\section{Authors' contributions}

$\mathrm{RD}$ performed the experiments on $\mathrm{ISH}, \mathrm{ICH}$ and drafted the manuscript. SY did the work on H.E. staining and ISH. JJ cloned the Stra8 CDNA and did the work on tissue distribution. TW and HS did the work on western blot. LZ did the work on bioinformatics' analysis. YZ and DW designed and directed the study and write the manuscript. All authors read and approved the final manuscript.

\section{Acknowledgements}

This work was supported by grants 31030063 from the National Natural Science Foundation of China; Grants 2012CB723205, 2011AA100404 and 2010 CB134405 from the National Basic Research Program of China; Grant 20090182110008 from the Specialized Research Fund for the Doctoral Program of Higher Education of China.

Received: 1 February 2013 Accepted: 15 May 2013 Published: 22 May 2013

\section{References}

1. Wilkins AS, Holliday R: The evolution of meiosis from mitosis. Genetics 2009, 181(1):3-12.

2. Bowles J, Koopman P: Retinoic acid, meiosis and germ cell fate in mammals. Development 2007, 134(19):3401-3411.

3. Bowles J, Koopman P: Sex determination in mammalian germ cells: extrinsic versus intrinsic factors. Reproduction (Cambridge, England) 2010, 139(6):943-958.

4. Koubova J, Menke DB, Zhou Q, Capel B, Griswold MD, Page DC: Retinoic acid regulates sex-specific timing of meiotic initiation in mice. Proc Natl Acad Sci U S A 2006, 103(8):2474-2479.

5. Li J, Liu Z, Wang D, Cheng CH: Insulin-like growth factor 3 is involved in oocyte maturation in zebrafish. Biol Reprod 2011, 84(3):476-486.

6. Menke DB, Page DC: Sexually dimorphic gene expression in the developing mouse gonad. Gene expression patterns: GEP 2002, 2(3-4):359-367.

7. Wright WW: New insights into the regulation of gametogenesis by retinoic acid. Biol Reprod 2010, 83(6):890-892.

8. Bowles J, Knight D, Smith C, Wilhelm D, Richman J, Mamiya S, Yashiro K, Chawengsaksophak K, Wilson MJ, Rossant J, et al: Retinoid signaling determines germ cell fate in mice. Science (New York, NY) 2006, 312(5773):596-600.

9. Trautmann E, Guerquin MJ, Duquenne C, Lahaye JB, Habert R, Livera G: Retinoic acid prevents germ cell mitotic arrest in mouse fetal testes. Cell cycle (Georgetown, Tex) 2008, 7(5):656-664.

10. Swain A: Sex determination: time for meiosis? The gonad decides. Current biology : CB 2006, 16(13):R507-R509.

11. Smith CA, Roeszler KN, Bowles J, Koopman P, Sinclair AH: Onset of meiosis in the chicken embryo; evidence of a role for retinoic acid. BMC Dev Biol 2008, 8:85.

12. Wallacides A, Chesnel A, Chardard D, Flament S, Dumond H: Evidence for a conserved role of retinoic acid in urodele amphibian meiosis onset. Developmental dynamics : an official publication of the American Association of Anatomists 2009, 238(6):1389-1398.

13. Anderson EL, Baltus AE, Roepers-Gajadien HL, Hassold TJ, de Rooij DG, van Pelt AM, Page DC: Stra8 and its inducer, retinoic acid, regulate meiotic initiation in both spermatogenesis and oogenesis in mice. Proc Natl Acad Sci U S A 2008, 105(39):14976-14980.

14. Han F, Liu ZH, Wu FR, Huang BF, Zhang WL, Zhou H, Wang DS: Involvement of retinoic acid and Cyp26 family genes in germ cell differentiation. Chinese Journal of Cell Biology 2008, 31:355-360.

15. Mark M, Jacobs H, Oulad-Abdelghani M, Dennefeld C, Feret B, Vernet N, Codreanu CA, Chambon P, Ghyselinck NB: STRA8-deficient spermatocytes 
initiate, but fail to complete, meiosis and undergo premature chromosome condensation. J Cell Sci 2008, 121(Pt 19):3233-3242.

16. Mi ML, Yang B, Xu SF, Zou T: [Stra8: a specifically expressed gene in premeiotic germ cells' transition from mitosis to meiosis]. Zhonghua nan ke $x$ ue = National journal of andrology 2009, 15(1):51-55.

17. Baltus AE, Menke DB, Hu YC, Goodheart ML, Carpenter AE, de Rooij DG, Page DC: In germ cells of mouse embryonic ovaries, the decision to enter meiosis precedes premeiotic DNA replication. Nat Genet 2006, 38(12):1430-1434.

18. Saito D, Morinaga C, Aoki Y, Nakamura S, Mitani H, Furutani-Seiki M, Kondoh $\mathrm{H}$, Tanaka M: Proliferation of germ cells during gonadal sex differentiation in medaka: Insights from germ cell-depleted mutant zenzai. Dev Biol 2007, 310(2):280-290.

19. Zhou Q, Li Y, Nie R, Friel P, Mitchell D, Evanoff RM, Pouchnik D, Banasik B, McCarrey JR, Small C, et al: Expression of stimulated by retinoic acid gene 8 (Stra8) and maturation of murine gonocytes and spermatogonia induced by retinoic acid in vitro. Biol Reprod 2008, 78(3):537-545.

20. Zhang XY, Jiao BW, Wu TL, Jin CB, Wang DS: Histological observation on gonadal sex differentiation in the Southern catfish, Silurus meridionalis. Chinese Journal of Zoology 2005, 40:41-48.

21. Childs AJ, Cowan G, Kinnell HL, Anderson RA, Saunders PT: Retinoic acid signalling and the control of meiotic entry in the human fetal gonad. PLoS One 2011, 6(6):e20249.

22. Griswold MD, Hogarth CA, Bowles J, Koopman P: Initiating meiosis: the case for retinoic acid. Biol Reprod 2012, 86(2):35.

23. Han R: [Pharmacological studies of N-4-(hydroxycarbophenyl) retinamide]. Zhongguo yi xue ke xue yuan xue bao Acta Academiae Medicinae Sinicae 1991, 13(2):101-107.

24. Lu XY, Yang B, Xu SF, Zou T: [STRA8 as a specific expression marker in postnatal male germ cells]. Zhonghua nan ke xue = National journal of andrology 2010, 16(2):161-165.

25. Tedesco M, La Sala G, Barbagallo F, De Felici M, Farini D: STRA8 shuttles between nucleus and cytoplasm and displays transcriptional activity. J Biol Chem 2009, 284(51):35781-35793.

26. Yamaguchi T, Kitano T: High temperature induces cyp26b1 mRNA expression and delays meiotic initiation of germ cells by increasing cortisol levels during gonadal sex differentiation in Japanese flounder. Biochem Biophys Res Commun 2012, 419(2):287-292.

27. Thompson JD, Gibson TJ, Plewniak F, Jeanmougin F, Higgins DG: The CLUSTAL_X windows interface: flexible strategies for multiple sequence alignment aided by quality analysis tools. Nucleic Acids Res 1997, 25(24):4876-4882.

28. Livak K, Schmittgen TD: Analysis of temporal gene expression data using real time quantitative PCR and the 2 (-Delta Delta C (T)) Method. Methods 2001, 25:402-408.

29. Wu FR, Zhou LY, Nagahama Y, Wang DS: Duplication and distinct expression patterns of two thrombospondin-1 isoforms in teleost fishes. Gene expression patterns: GEP 2009, 9(6):436-443.

doi:10.1186/1471-2199-14-11

Cite this article as: Dong et al:: Characterization of Stra8 in Southern catfish (Silurus meridionalis): evidence for its role in meiotic initiation BMC Molecular Biology 2013 14:11.

\section{Submit your next manuscript to BioMed Central and take full advantage of:}

- Convenient online submission

- Thorough peer review

- No space constraints or color figure charges

- Immediate publication on acceptance

- Inclusion in PubMed, CAS, Scopus and Google Scholar

- Research which is freely available for redistribution 